La

Révolution

française

\section{La Révolution française}

Cahiers de l'Institut d'histoire de la Révolution française

13 | 2018

Pratiques et enjeux scientifiques, intellectuels et politiques de la traduction (vers 1660-vers 1840)

\title{
Suède, Europe, Japon : Le botaniste Carl Peter Thunberg sur le marché international
}

\section{Marie-Christine Skuncke}

\section{CpenEdition}

\section{Journals}

Édition électronique

URL : http://journals.openedition.org/lrf/1928

DOI : $10.4000 /$ Irf. 7928

ISSN : 2105-2557

Éditeur

IHMC - Institut d'histoire moderne et contemporaine (UMR 8066)

Référence électronique

Marie-Christine Skuncke, « Suède, Europe, Japon : Le botaniste Carl Peter Thunberg sur le marché international », La Révolution française [En ligne], 13 | 2018, mis en ligne le 22 janvier 2018, consulté le 03 mai 2019. URL : http://journals.openedition.org/lrf/1928 ; DOI : 10.4000/lrf.1928

Ce document a été généré automatiquement le 3 mai 2019.

(c) La Révolution française 


\title{
Suède, Europe, Japon : Le botaniste Carl Peter Thunberg sur le marché international
}

\author{
Marie-Christine Skuncke
}

1 Disciple de Linné, le botaniste et médecin suédois Carl Peter Thunberg (1743-1828), est le seul savant européen à avoir visité et décrit le Japon au xvIII ${ }^{\mathrm{e}}$ siècle $^{1}$. Ses ouvrages, traduits en plusieurs langues, ont connu une large diffusion en Europe et ont eu un retentissement au Japon. On l'a surnommé « le père de la botanique japonaise ». Le cas individuel de Thunberg permet d'éclairer les pratiques de la traduction entre langues européennes à la fin du XVIII ${ }^{\mathrm{e}}$ siècle, et aussi d'élargir l'horizon vers l'Asie.

2 Après de solides études de médecine et d'histoire naturelle à l'université d'Uppsala, puis à Paris, Thunberg entreprend en 1772 un long voyage vers l'Extrême Orient. Il a pour mission d'envoyer des arbres et arbustes vivants du Japon à Amsterdam, au Jardin botanique (Hortus Medicus) et à trois riches amateurs de plantes, membres de l'oligarchie au pouvoir dans la cité. L'empire japonais est fermé aux Européens au XvIII ${ }^{\mathrm{e}}$ siècle, à l'exception de la Compagnie néerlandaise des Indes orientales, et Thunberg voyage en qualité de chirurgien au service de la compagnie. En cours de route, il visite des territoires de l'empire colonial néerlandais : la province du Cap en Afrique du Sud, où il demeure trois ans, Java et Ceylan. Il passe quinze mois au Japon en 1775-1776, cantonné la plupart du temps dans la minuscule île de Deshima, à Nagasaki, résidence des marchands hollandais. Partout, même au Japon, grâce à ses échanges avec des interprètes à Nagasaki et des médecins de cour à Edo (aujourd'hui Tokyo), il assemble de vastes collections sur lesquelles il va baser sa carrière : plantes surtout, insectes, monnaies, livres, etc.

De retour en Suède en 1779, Thunberg fait une ascension fulgurante à l'université d'Uppsala. En cinq ans, il passe de simple démonstrateur de botanique à titulaire de la chaire de médecine et de botanique - celle de son maitre Linné - qu'il occupera pendant quarante-quatre ans, de 1784 à sa mort ${ }^{2}$.

4 Thunberg publie énormément, en suédois et en néo-latin; sa bibliographie comprend près de 580 titres $^{3}$. Il s'agit en grande partie de textes scientifiques, descriptions de 
familles ou d'espèces nouvelles de plantes ou d'insectes selon le système de Linné, mais aussi de textes destinés à un public plus large, un récit de voyage surtout, publié en suédois en quatre volumes de 1788 à 1793. Andreas Önnerfors a étudié en détail la genèse des deux traductions allemandes de ce livre ${ }^{4}$.

La diffusion des textes de Thunberg soulève plusieurs questions. Dans quelle langue choisit-il de publier ses ouvrages? En latin? Ou en langue vernaculaire qui nécessite des traductions? Pour celles-ci, dans quelle mesure l'auteur est-il capable de contrôler la version de ses œuvres qui est publiée à l'étranger, et quels rôles respectifs jouent les traducteurs, les rédacteurs et les éditeurs-libraires? Sur le plan textuel, quelles sont les relations entre original et traductions? Trois temps seront abordés dans cette étude : d'abord un aperçu de la diffusion européenne des monographies scientifiques et articles de Thunberg, puis une présentation de celle de son récit de voyage, où nous nous concentrerons sur les versions françaises, et enfin sera évoquée sur une publication japonaise au début du XIX siècle.

\section{Monographies scientifiques et articles}

6 Le néo-latin demeure vivace dans le domaine de la botanique. C'est dans cette langue que Linné a élaboré sa nomenclature et écrit ses œuvres majeures, et Thunberg suit l'exemple de son maître ${ }^{5}$. Il rédige en latin sa monographie sur les plantes du Japon, la Flora Japonica (1784), publiée stratégiquement à Leipzig pour atteindre le public savant européen, puis au début du xix siècle! - la Flora Capensis consacrée à l'Afrique du Sud, éditée successivement à Uppsala, Copenhague et Stuttgart (1807-1823) ${ }^{6}$. Ces œuvres sont diffusées en Europe sans passer par la traduction.

7 Tantôt en latin, tantôt en suédois, Thunberg écrit des quantités d'articles pour les mémoires de sociétés savantes, réglant le choix de la langue sur la pratique des sociétés auxquelles il s'adresse - par exemple, le suédois pour l'Académie des sciences de Stockholm, mais le latin pour l'Académie Impériale des Sciences de Saint-Pétersbourg. Outre la botanique et la zoologie, les textes touchent aussi l'économie : ainsi une étude sur la production de la cannelle à Ceylan, une autre sur la numismatique (un discours sur les monnaies japonaises), etc. ${ }^{7}$ De nombreux articles sont traduits vers d'autres langues : néerlandais, allemand, anglais, français, russe. Quelques exemples le démontrent :

Les Pays-Bas d'abord, avec deux traductions qui s'inscrivent dans un jeu d'échanges entre Thunberg et ses patrons et collègues. L'un des riches collectionneurs d'Amsterdam auxquels le Suédois a envoyé des plantes rares pendant son voyage, Jean Deutz, remercie Thunberg de ses services en le faisant élire membre de la Société des sciences à Haarlem, dont il est directeur. En même temps, Deutz demande au naturaliste Martinus Houttuyn de traduire un article de Thunberg sur les cycas pour les mémoires de la Société de Haarlem. Houttuyn traduit en néerlandais le manuscrit latin de Thunberg, non sans manifester son irritation contre son écriture minuscule, et, ainsi, la publication originale a lieu en néerlandais en $1782^{8}$. Le botaniste suédois, en signe de reconnaissance pour les services de Houttuyn, donne son nom à une plante japonaise, la Houttuynia. Houttuyn, à son tour, remercie Thunberg en le faisant élire membre de la Société des sciences de Vlissingen, et il publie dans les mémoires de cette société une traduction néerlandaise de l'article de Thunberg sur la cannelle, dont l'original avait paru en suédois dans les mémoires de l'Académie des sciences de Stockholm?. 
9 La Grande-Bretagne ensuite. Dans les mémoires de la Royal Society à Londres, les Philosophical Transactions, on lit en 1780 un article impeccablement imprimé en suédois, un «bref extrait d'un journal tenu par le docteur Thunberg » pendant son voyage et son séjour au Japon ; les Transactions donnent une traduction anglaise en appendice ${ }^{10}$. Il s'agit d'un texte non spécialisé, un chapelet de détails exotiques sur le Japon : le costume, les coiffures, les parasols... Comment se fait-il que le vénérable périodique britannique publie un article en version originale suédoise avec une traduction anglaise ? Encore une fois, les textes s'inscrivent dans un jeu d'échanges au sein des réseaux de Thunberg. Le personnage principal est le naturaliste Joseph Banks, puissant président de la Royal Society. Il est entouré de collaborateurs suédois, Daniel Solander, élève de Linné, et Jonas Dryander, qui a été condisciple de Thunberg à Uppsala. Thunberg voit en Banks un patron potentiel. Il lui rend visite à Londres à son retour du Japon, lui fait la cour, lui envoie des plantes japonaises rarissimes. Banks, de son côté, publie des articles du voyageur dans les Transactions. En 1780, les collaborateurs suédois du président de la Royal Society ont dû veiller à l'impression du texte suédois et à la traduction anglaise.

10 Avec la parution de la version anglaise dans les Transactions, le petit article de Thunberg sur le Japon est soumis aux mécanismes du marché. Une traduction française dans le Journal Encyclopédique, en 1781, est reprise la même année dans l'Esprit des journaux. Une traduction allemande, fondée elle aussi sur la version anglaise, paraît dans l'almanach de poche de Göttingen pour l'année 1782, imprimé fin $1781^{11}$. En deux ans, passant du format in-quarto pour les Philosophical Transactions à in-octavo pour les journaux de langue française et in-16 pour l'almanach de Göttingen, les nouvelles de Thunberg sur le Japon atteignent le public anglophone, francophone et germanophone.

\section{Le récit de voyage et ses transformations}

11 Thunberg tarde à écrire son récit de voyage, Resa uti Europa, Africa, Asia ${ }^{12}$. Le premier tome (1788) relate ses voyages en Europe et en Afrique du Sud, le second (1789) en Afrique du Sud et à Java. En 1791 paraît enfin un volume entièrement consacré au Japon, le troisième tome, quinze ans après la visite de Thunberg. Le quatrième tome, en 1793, comprend la suite de son séjour au Japon et son retour en Europe par Java et Ceylan. Pour les lecteurs européens, l'ouvrage offre donc un attrait majeur: il n'existe pas de description de première main du Japon depuis la visite de l'Allemand Engelbert Kaempfer dans les années $1690^{13}$. Sur l'empire nippon et sur les autres pays qu'il traverse, Thunberg apporte des observations de tous ordres : botaniques et zoologiques, mais aussi météorologiques, linguistiques, ethnographiques, etc. 


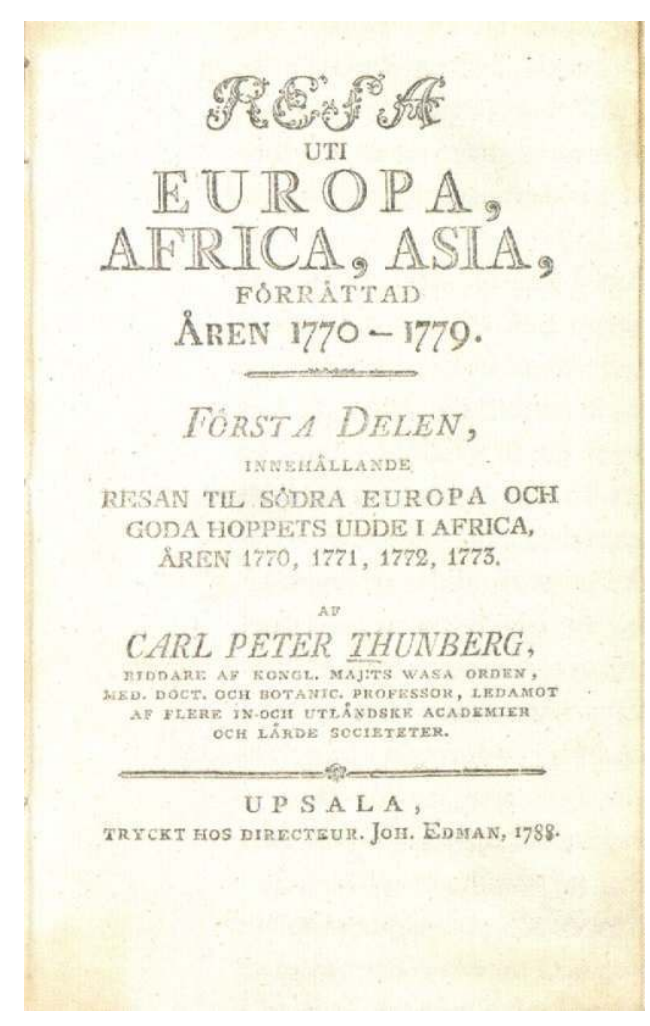

Le récit de voyage de Thunberg, version originale, page de titre :

Carl Peter Thunberg, Resa uti Europa, Africa, Asia, Förrättad Åren 1770 - 1779,

vol. 1, Upsala [à compte d'auteur], 1788.

Source : Uppsala universitetsbibliotek.

Cependant, son livre, publié à compte d'auteur sans passer par un éditeur professionnel, présente des faiblesses. La structure est confuse: l'auteur oscille entre récit chronologique et sections thématiques; il recopie souvent des textes qu'il a publiés antérieurement, et les redites sont nombreuses. Thunberg accumule les faits, mais ne donne guère d'analyses. L'illustration est pauvre, bien inférieure à celle du récit de Kaempfer, dont une édition allemande est parue dans les années $1770^{14}$. Les gravures sont peu nombreuses et leur qualité laisse à désirer - l'université d'Uppsala ne dispose pas de graveur professionnel. Le troisième tome, celui qu'on attendait, sur le Japon, ne contient pas une seule planche, le quatrième offre quatre planches seulement représentant des objets japonais.

Le récit de voyage, écrit Roger Chartier, est «l'un des genres les plus conquérants » au $\mathrm{XVIII}^{\mathrm{e}}$ siècle $^{15}$. Il n'est donc pas étonnant que le livre de Thunberg soit rapidement traduit. En l'espace de quatre ans, de 1792 à 1796, on voit paraître deux traductions allemandes, une traduction anglaise qui connaît plusieurs éditions, et deux traductions françaises. En Allemagne comme à Londres et à Paris, deux équipes rivales sont en compétition. Sur le marché allemand, comme l'a montré Andreas Önnerfors, le traducteur Christian Heinrich Groskurd à Greifswald et l'éditeur berlinois Johann Karl Philipp Spener sont talonnés par le naturaliste Johann Reinhold Forster à Halle, rédacteur d'une collection de récits de voyages, son traducteur Kurt Polycarp Sprengel et leur éditeur berlinois Christian Friedrich Voss ${ }^{16}$. À Londres, le traducteur Charles Hopson, un médecin, fait la course avec une équipe d'amateurs appartenant à un réseau swedenborgien ; les détails sont obscurs, mais il semble que Hopson l'ait emporté ${ }^{17}$. À Paris, enfin, un projet centré autour du 
libraire Benoît d'André est en concurrence avec une publication éditée par le libraire Jean-Jacques Fuchs.

Devant le texte assez informe de Thunberg, les traducteurs et rédacteurs adoptent plusieurs stratégies :

- Réduction du texte à un seul volume. C'est le cas de la version allemande de Forster, et de la première version française, publiée par Fuchs.

- Traduction du livre entier en quatre volumes (ou deux volumes doubles), mais avec une structure plus nette. C'est ce qui se passe pour l'édition allemande de l'équipe GroskurdSpener et pour la traduction anglaise.

- Publication en quatre volumes, mais réécriture et expansion du texte original. C'est le cas de la deuxième version française, éditée par Benoît d'André (ou Dandré).

Il est intéressant d'examiner dès lors les deux versions françaises, le texte abrégé paru chez Fuchs en 1794, et, surtout, la version étendue publiée par d'André en $1796^{18}$. Pour cette dernière, une série de quarante-deux lettres manuscrites de Benoît d'André à Thunberg entre 1791 et 1797, découvertes en 2013 à la Bibliothèque universitaire d'Uppsala par l'auteur de cet article, permet de reconstituer en grande partie la genèse $\mathrm{du}$ livre ${ }^{19}$. Pour le texte édité par Fuchs, par contre, l'unique source dont nous disposons est le volume imprimé.

En novembre 1791, le libraire-éditeur d'André offre à Thunberg en termes flatteurs de publier une traduction française de son livre, incité par la « celebrité de [son] Nom » et son rang de "savant du premier ordre ». Grâce à «l'Universalité de Notre Langue », lui dit-il, ses ouvrages seraient bientôt répandus « dans le Monde entier ». D'André propose à Thunberg de faire traduire son récit à Uppsala, «meme en mauvais français ", par une personne qui sera rémunérée par le libraire. Le texte sera ensuite "perfection[né] » à Paris par « un savant, et habile Botaniste» (il ne révèle pas son nom). Les planches de l'original seront gravées à Paris par « les plus habiles Artistes ». Il y aura une dédicace à Thunberg et un portrait de lui en tête de l'ouvrage, aux frais du libraire. D'André demande au Suédois d'envoyer sa correspondance soit par la poste, soit, de préférence, par l'ambassade de France à Stockholm, et de l'adresser au « Departement de la Suède aux Bureaux des Affaires étrangères ». Il se présente comme "Libraire de la Société Royale de Medecine de Paris », et se réclame de l'autorité du secrétaire de celle-ci, l'anatomiste Vicq d'Azyr, médecin du roi et de la reine ${ }^{20}$.

Les lettres de d'André en 1791-1792 suggèrent qu'il a accès, pour transmettre sa correspondance, à des personnes haut placées, probablement grâce à son protecteur Vicq d'Azyr, et dispose de moyens financiers non négligeables. Cependant, en pleine tourmente révolutionnaire, les changements institutionnels se succèdent. À plusieurs reprises, d'André donne à Thunberg de nouvelles instructions concernant le ministère et le ministre auxquels il devra adresser ses envois. En Suède, l'ambassade de France à Stockholm ferme, et Thunberg doit désormais passer par le consul de France à Göteborg, Delisle, ami de d'Andréê. Malgré les bouleversements politiques, pourtant, les affaires continuent; pendant l'année 1793, d'André n'écrit pas moins de dix-huit lettres à Thunberg. Il évite de parler politique, mise à part une allusion en septembre 1792 aux «mouvements terribles» qui secouent le pays ${ }^{22}$. La Société Royale de Médecine est dissoute en août 1793. En décembre de cette année, le libraire informe Thunberg que les lettres doivent désormais lui être envoyées personnellement au nom de «Citoyen Dandré Libraire, Rue du Cimètiere [sic] St André des Arts »; il a modifié l'orthographe de son nom, adoptant la forme plébéienne « Dandré » ${ }^{23}$. 

domicilié en Suède pour traduire son ouvrage, un certain Villeneuve. D'André envoie à Thunberg cent cinquante riksdaler suédoises à verser au traducteur pour les trois premiers volumes, une somme considérable. Il reçoit le premier volume de la traduction en janvier 1793 et la trouve abominable: Villeneuve ne sait pas un mot de français, Thunberg ne doit pas lui donner un liard de plus! D'André va être obligé de «faire rédiger toute la traduction à neuf» par son rédacteur, à grands frais pour lui-même. Thunberg recrute un deuxième traducteur, qui meurt, puis un troisième, qui termine le dernier volume. Un manuscrit est longtemps perdu dans les bureaux d'un ministère, mais finit par être retrouvé. En juin 1794, d'André a enfin reçu de Suède la traduction complète ${ }^{24}$.

d'André attache une grande importance à l'illustration visuelle, mais il se heurte à des difficultés. Premier problème, le portrait de l'auteur. Le professeur suédois ne possède pas d'image de lui-même - à la différence de son maître Linné, Thunberg n'a pas de relations dans le monde artistique suédois. Il envoie à Paris deux dessins que d'André trouve très mal exécutés, « même contre les principes de l'art ». Le libraire les transmet à son «grand, et habile Dessinateur $»^{25}$. Celui-ci, explique d'André deux mois plus tard, a choisi « le milieu » entre les deux dessins envoyés par Thunberg, «faisant voir dans vos yeux le caractère savant qui les anime $»^{26}$.

Deuxième problème, les planches. Celles-ci font l'objet de longues discussions entre Thunberg, d'André et son rédacteur, dont le nom est enfin révélé par le libraire en 1794 : il s'agit de Jean-Baptiste Lamarck, qui a obtenu l'année précédente une chaire de zoologie au Muséum d'histoire naturelle ${ }^{27}$. D'André s'est procuré les planches de l'original suédois par l'intermédiaire du traducteur allemand de Thunberg, Groskurd, et les trouve «très mal gravées ». Le libraire parle avec dédain de "vos médiocres artistes »; il réclame des dessins, ou au moins des descriptions qui puissent servir de directives aux dessinateurs et graveurs parisiens ${ }^{28}$. Avant tout, il faut davantage de planches sur le Japon, à la fois dans le troisième volume, qui n'en contient pas, et dans le quatrième. D'André désire des illustrations botaniques et zoologiques inédites - «quelqu'oiseau rare, et inconnu ", quelque papillon - accompagnées de détails scientifiques précis, par exemple «l'endroit, et le nom du terroir » où Thunberg a cueilli ses plantes. "Ces descriptions, et figures plaisent infinim[en]t aux amateurs d'histoire naturelle ", explique-t-il ${ }^{29}$. Le libraire vise un produit haut de gamme pour un lectorat instruit et aisé. Le rédacteur Lamarck, par l'intermédiaire de d'André, demande également une carte géographique du Japon et des «objets œeconomiques", plantes pour la teinture, trempe de l'acier, porcelaine... Thunberg envoie des propositions supplémentaires : illustrations botaniques - ainsi un thuya japonais (Thuja dolabrata) -, dessins d'objets japonais - perruques, miroir -, etc. ${ }^{30}$. En été 1794, la publication du récit de voyage semble proche. Mais, le $1^{\mathrm{er}}$ août 1794 , quelques jours après la chute de Robespierre, d'André tombe gravement malade et l'entreprise est retardée ${ }^{31}$.

21 Le libraire parisien correspond avec des collègues dans le monde de l'édition germanophone, et il collabore avec Groskurd, le traducteur de Thunberg à Greifswald. En mai 1792, d'André avait envoyé des nouvelles alarmantes à Uppsala : il avait eu vent d'une entreprise rivale à Paris, une traduction française fondée sur une version abrégée allemande en cours de préparation à Berlin (c'est-à-dire la version de Forster). Pour court-circuiter les projets concurrents à Berlin et à Paris, d'André avait demandé à 
Thunberg de retarder la parution de l'édition originale du quatrième volume de son récit, afin que le dernier volume en suédois, la traduction allemande de Groskurd et la version française de d'André puissent être publiées simultanément ${ }^{32}$. Hélas pour le libraire, ceci s'avère impossible, et la traduction française rivale sort en 1794, avant la sienne.

Le Voyage en Afrique et en Asie, publié en un volume in octavo par le libraire Fuchs, est un produit anonyme. D'après la préface, le traducteur avait commencé par travailler sur l'original suédois, mais, trouvant le style de l'auteur insoutenablement prolixe, il a choisi de suivre la version allemande abrégée de Forster, en conservant toutefois certaines parties de l'original omises par ce dernier. La page de titre présente l'ouvrage comme «servant de suite au voyage de D. Sparmann [sic]» - il s'agit du naturaliste suédois Anders Sparrman, disciple lui aussi de Linné, qui a publié un récit de ses voyages en Afrique du Sud. De fait, il n'existe aucune relation séquentielle entre les textes de Sparrman et de son collègue Thunberg, mais les deux auteurs sont des voyageurs suédois et le livre de Sparrman a été traduit en français avant celui de Thunberg ${ }^{33}$. Le traducteur anonyme interpole dans le corps du texte un passage tiré de l'Allemand Kaempfer sur la fabrication du papier au Japon, ainsi que des observations sur le Japon extraites du Voyage en Sibérie du botaniste Johann Gottlieb Georgi ${ }^{34}$. L'édition française de 1794, amalgamant des textes sur les mêmes thèmes mais sans rapports historiques, est un exemple de la logique du marché dans la production des récits de voyages.

L'entreprise de d'André, cependant, se poursuit. Depuis sa maladie en août 1794, le libraire n'envoie à Thunberg que des lettres sporadiques, d'une écriture déformée par la paralysie. En septembre 1795, il annonce que le Voyage de Thunberg va paraitre d'ici la fin du mois ; deux mois plus tard, il écrit qu'il y aura une deuxième édition ${ }^{35}$. La firme Dandré (avec la nouvelle orthographe) publie en effet deux éditions du livre de Thunberg, l'une en quatre volumes in octavo, l'autre en deux volumes in quarto. Le titre est le même, Voyages de C. P. Thunberg, au Japon, par le Cap de Bonne-Espérance, les Isles de la Sonde, \&c, ainsi que la date : «An IV (1796) ». Exceptées les différences de pagination et de mise en page dues à la différence de format, les deux éditions paraissent identiques ${ }^{36}$. En tête du premier volume, on trouve un frontispice correspondant au projet original de d'André : un portrait gravé de Thunberg, portant le costume national suédois et l'Ordre de Vasa, dans une ovale entourée de deux plantes nommées en son honneur, la Thunbergia capensis et la Thunbergia javanica ; sous le portrait, une dédicace en latin, «À Carl Peter Thunberg, Professeur de Botanique à Uppsala, Chevalier de l'Ordre de Vasa, homme de mérite ${ }^{37}$ ». La page de titre, par contre, réserve une surprise. Lamarck, qui figurait en qualité de « rédacteur » dans les lettres du libraire jusqu'à sa maladie, est relégué au deuxième rang, en tant que responsable de « la partie d'Histoire naturelle». Le premier rang revient à « L. LANGLES", qui est présenté comme traducteur, rédacteur, et auteur de "notes considérables sur la Religion, le Gouvernement, le Commerce, l'Industrie et les Langues de ces différentes contrées, particulièrement sur le Javan et le Malai ». 


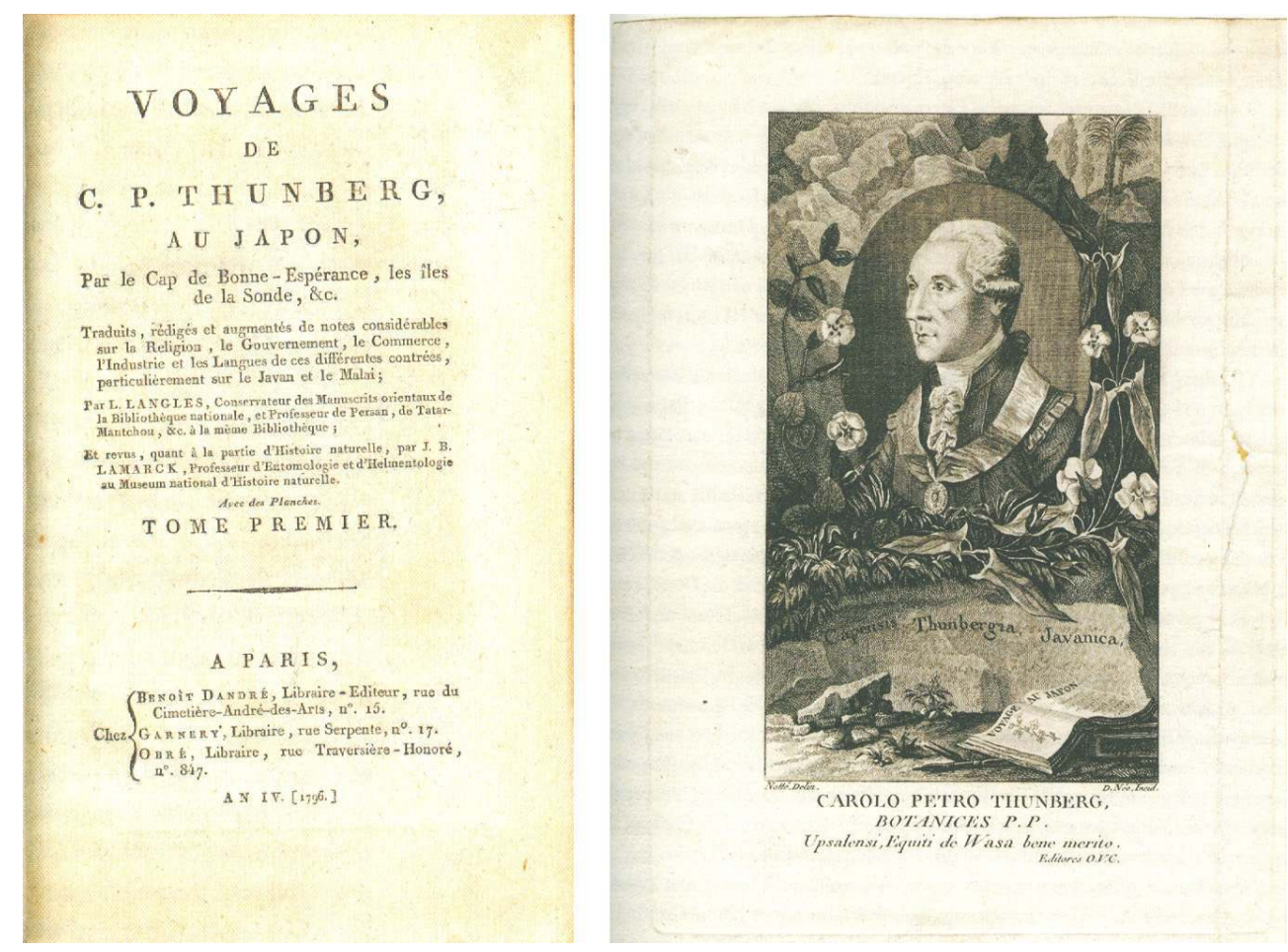

À gAUCHE : Le récit de voyage en version française, page de titre : Voyages de C. P. Thunberg au Japon, Par le Cap de Bonne-Espérance, les îles de la Sonde, \&c., [réd.] L. Langlès \& J. B. Lamarck, vol. 1, Paris, Benoît Dandré, an IV [1796], édition in octavo.

À DRoITE : Thunberg, Voyages..., vol. 1, Paris, Benoît Dandré, an IV [1796] : frontispice. Source : Uppsala universitetsbibliotek.

Alors que Lamarck est naturaliste, Louis-Mathieu Langlès est linguiste, conservateur des manuscrits orientaux à la Bibliothèque nationale. En 1795, il a fondé dans le cadre de celle-ci l'École des langues orientales. Il a dû entrer dans l'équipe de d'André entre le $1^{\mathrm{er}}$ août 1794, quand le libraire est tombé malade, et octobre 1795, date indiquée par Langlès dans sa "Préface du Rédacteur». Les sources manquent quant à la raison de son implication dans le projet de traduction, mais l'ouvrage imprimé montre qu'il a joué un rôle prépondérant ${ }^{38}$. Dans sa préface, il se pose en "traducteur» - affirmation contestable puisqu'il a accès à la traduction française complète envoyée par Thunberg et surtout en « rédacteur $»^{39}$. Ce dernier titre est hautement justifié. Sous la direction de Langlès, l'original plutôt maladroit de Thunberg est transformé. Le corps du texte est entièrement restructuré, divisé en "Parties » et "Chapitres » qui suivent en partie la traduction allemande de Groskurd. En même temps, on assiste à une expansion de l'original. Des notes en bas de page par Langlès, et par Lamarck pour l'histoire naturelle, expliquent, commentent et contredisent les assertions de Thunberg dans le corps du texte. Langlès ajoute des sections de sa plume dans les chapitres sur Java, en particulier une étude de l'ancienne langue "javane ", distincte du malai dont traite Thunberg, avec un bref vocabulaire. Il met en appendice une adaptation du discours du Suédois sur les monnaies japonaises ${ }^{40}$.

Le récit original de Thunberg met l'accent sur ses expériences personnelles et contient peu de références à d'autres auteurs. Langlès, par contre, place le texte du professeur suédois dans un contexte global et adopte une perspective comparatiste. Il rassemble des informations sur l'Asie à partir de sources à la fois asiatiques et européennes, avec une compétence linguistique qui devait être unique en France à l'époque. Il se repose par 
exemple sur les mémoires en néerlandais de l'Académie des sciences de Batavia (l'actuelle Jakarta, capitale de l'empire colonial néerlandais), sur ceux en anglais de la Société asiatique de Calcutta, et sur d'« anciennes Lettres latines » de missionnaires. Il présente ses sources dans une bibliographie annotée de près de cinquante pages à la fin du dernier volume, une mine d'informations ${ }^{41}$. Sa préface commence par une comparaison entre la Chine, " exclusivement livrée aux lettres et aux sciences spéculatives» et devenue pour cette raison la proie d'autres pays, et le Japon, resté fidèle à ses anciennes lois, actif, inconquis et libre. Il digresse sur l'histoire comparée des religions, suggérant une « identité [...] entre les cultes anciens et modernes de l'Asie et de l'Europe " $^{42}$.

Langlès ne perd pas de vue les intérêts de la jeune République française. L'alliance de celle-ci avec les Hollandais (conséquence de la création de la République batave en 1795) pourrait, dit-il, ouvrir pour la France l'accès au commerce avec le Japon, jusqu'alors réservé à la Compagnie néerlandaise ${ }^{43}$. L'édition de Langlès illustre deux tendances observées par Jean-Luc Chappey et Virginie Martin dans les traductions françaises de l'époque ${ }^{44}$. D'une part, le traducteur confère à l'ouvrage original une dimension universelle, dans un processus à la fois de « circulation des savoirs en Europe et [de] leur thésaurisation en France ", selon les deux auteurs. D'autre part, le texte original est républicanisé. Langlès affirme que l'enseignement des langues orientales - sa propre branche - permettrait d'étendre le commerce français en Asie et, en même temps, d'y diffuser les valeurs de la République. Dans une note à propos d'un passage chez Thunberg sur le roi de Suède Gustave III, le rédacteur loue l'assassin de celui-ci, «l'intrépide et immortel Ankastrom ${ }^{45}$ » (le nom correct est Anckarström).

Les illustrations constituaient une préoccupation majeure pour d'André. Elles sont à la fois plus nombreuses et de meilleure qualité technique que dans l'original. En plus du frontispice, le libraire a fait graver une nouvelle vignette de la vue du Cap de Bonne Espérance au début de l'ouvrage. Toutes les planches de l'édition suédoise sont reproduites, et le dernier volume, dans l'édition in-octavo, ne contient pas moins de onze planches nouvelles : quatre d'entre elles représentent des objets japonais et cingalais, entre autres les perruques et le miroir japonais envoyés par Thunberg; sept sont consacrées à l'histoire naturelle, notamment une plante japonaise, le Lobelia radicans, mais pas le thuya dont il avait été question dans la correspondance. De plus, d'André reproduit les planches du discours sur les monnaies japonaises.

Thunberg, dans une lettre au jardinier André Thouin à Paris en 1796, se plaint que son Voyage en traduction française est « bien vitieux, avec beaucoup d'errata et de meprises ${ }^{46}$ ». Les interventions de Lamarck ont dû particulièrement le contrarier. À l'époque, celuici n'a pas encore publié les travaux zoologiques où il formule ses hypothèses sur le transformisme, mais c'est un botaniste renommé - et il appartient au camp anti-linnéen en France. Il a publié une Flore françoise, il est responsable de plusieurs volumes sur la botanique dans l'Encyclopédie méthodique de Panckoucke ${ }^{47}$. Pour la traduction française de Thunberg, ses interventions dans les notes concernent des questions de nomenclature : il donne les noms scientifiques latins des plantes et animaux mentionnés en langue vernaculaire dans le corps du texte, et il discute - et souvent corrige - les observations de l'auteur suédois. Il ajoute aussi des descriptions scientifiques en français des animaux et plantes représentés dans les planches d'histoire naturelle du dernier volume ${ }^{48}$.

29 Au total, le récit de voyage de Thunberg est métamorphosé dans la version française de d'André - Langlès - Lamarck : structure nette, enrichissement de l'illustration visuelle, révision de l'information botanique et zoologique. L'ouvrage français ouvre des 
perspectives sur l'histoire des civilisations, en même temps qu'il s'inscrit dans la promotion de la République française. Bien que Thunberg et son éditeur aient mené une correspondance serrée pendant deux ans et demi, l'influence de l'auteur sur le résultat final est minime. Elle est nulle en ce qui concerne l'édition française parue chez Fuchs en 1794. Pourtant, ce sont les traductions - en français, en allemand, en anglais - qui font connaître le récit du Suédois sur le marché international. Notons que, si des équipes rivales sont en concurrence dans trois capitales européennes, on assiste aussi à une collaboration à travers les frontières. En pleine Révolution française, un libraire parisien envisage une publication simultanée du récit de Thunberg à Uppsala, Berlin et Paris.

\section{Retour au Japon}

Tournons-nous, pour terminer, vers le Japon. Près d'un demi-siècle après la visite de Thunberg, un chirurgien militaire allemand au service des Pays-Bas, Philipp Franz von Siebold, débarque à Nagasaki en 1823. Il apporte dans ses bagages la Flora Japonica de Thunberg ainsi que, probablement, son récit de voyage dans l'édition française de 1796.

$31 \mathrm{Au}$ début du XIX $\mathrm{X}^{\mathrm{e}}$ siècle, les élites intellectuelles, et aussi les autorités japonaises, manifestent un intérêt croissant pour la «science hollandaise ", c'est-à-dire les sciences occidentales, en japonais «Rangaku »" Von Siebold est autorisé à ouvrir une école de médecine près de Nagasaki. L'un de ses élèves, un brillant jeune botaniste originaire de Nagoya, se nomme Itō Keisuke. Lorsque celui-ci termine ses études chez von Siebold en 1828, l'Allemand lui donne en cadeau d'adieu la Flora Japonica. L'année suivante, Itō Keisuke, à 26 ans, publie à Nagoya une adaptation très libre du livre de Thunberg, le Taisei Honzō Meiso, synthèse de connaissances botaniques européennes et extrêmes-orientales. Itō Keisuke deviendra un patriarche de la botanique japonaise (il mourra en 1901 à 98 ans), et les travaux de Thunberg constitueront une « référence fondamentale » lorsqu'un Département de botanique sera créé à l'Université impériale de Tokyo après la Restauration Meiji. Le Taisei Honzō Meiso contient un portrait de Thunberg, gravé sur cuivre et non sur bois comme c'est la coutume $e^{50}$. Ce portrait, qui fait connaitre les traits du Suédois aux lecteurs japonais, est une reproduction méticuleuse du frontispice de l'édition française de 1796, fruit des hypothèses d'un dessinateur et d'un graveur parisiens à partir de deux médiocres dessins suédois : Thunberg tel qu'il devrait l'être.

\section{Conclusion}

Il est temps de revenir aux questions posées au début de cet article. Chez le botaniste Thunberg, le choix de la langue est lié, d'une part, au genre - néo-latin pour les monographies scientifiques, suédois pour le récit de voyage - et, d'autre part, aux pratiques des revues qui publient ses articles - par exemple latin pour les Actes de l'Académie impériale des sciences à Saint-Pétersbourg, mais aussi suédois et anglais pour les Philosophical Transactions à Londres en 1780 et néerlandais pour des revues de sociétés savantes aux Pays-Bas. Ces derniers cas nécessitent des traductions, du suédois ou du latin, qui s'inscrivent dans le cadre d'échanges de services au sein des réseaux scientifiques de Thunberg; l'auteur peut compter sur la fidélité des versions de ses collègues. 

Europe. L'ouvrage est traduit en allemand, anglais et français. Il ne l'est pas en néerlandais, bien que l'auteur traite des colonies néerlandaises d'Afrique et d'Asie, du comptoir de Deshima et de la mission néerlandaise à Edo. Pour les versions françaises, nous venons de le voir, l'influence de l'auteur sur le résultat final est minime ou nulle. Dans le cas de l'édition de 1796, dont les sources permettent de suivre en détail la genèse, les traducteurs jouent un rôle secondaire; nous ne connaissons même pas le nom de la personne qui a complété le travail de traduction en Suède. L'initiative de la publication vient du libraire parisien d'André, et ce sont les rédacteurs, Lamarck et, surtout, Langlès, qui ont déterminé la structure et le contenu de l'ouvrage publié. Pour les versions anglaise et allemandes, les sources permettent d'affirmer que le contrôle de l'auteur est nul dans deux cas : d'une part la traduction londonienne, d'autre part l'édition berlinoise en un volume, où le rédacteur Johann Reinhold Forster a joué un rôle prépondérant. Dans un cas seulement, l'édition berlinoise en deux volumes doubles, Thunberg a pu suivre régulièrement le processus de publication grâce à sa correspondance avec le traducteur allemand Groskurd. La proximité géographique et politique a dû faciliter les contacts : la ville de Greifswald où demeure Groskurd fait partie du royaume de Suède ${ }^{51}$.

traductions, celle de Groskurd et la version anglaise, suivent de près le texte suédois de Thunberg, l'original est transformé dans les trois autres traductions. Le cas du botaniste suédois suggère que le récit de voyage, dans l'Europe du XviII ${ }^{e}$ siècle, est un texte malléable qui peut être comprimé ou élargi à volonté, au gré des rédacteurs et éditeurs. Il y aurait ici matière à une enquête systématique sur les traductions de récits de voyage en Europe. Il faut noter, pour terminer, la transformation sur le sol japonais de l'œuvre scientifique maîtresse de Thunberg, la Flora Japonica, au début du XIX ${ }^{\mathrm{e}}$ siècle. Loin d'être un imitateur passif, le jeune auteur du Taisei Honzō Meiso refond l'ouvrage en 1829 à la lumière des derniers progrès de la science européenne et extrême-orientale.

\section{NOTES}

1. Le présent article est basé sur ma monographie Carl Peter Thunberg, Botanist and Physician: Career-Building Across the Oceans in the Eighteenth Century, Uppsala, Swedish Collegium for Advanced Study, 2014. Le livre a été écrit dans le cadre d'un projet financé par la fondation Riksbankens Jubileumsfond à Stockholm, "Carl Peter Thunberg, Europe and Japan », auquel a également participé Andreas Önnerfors. Je tiens à remercier le Swedish Collegium for Advanced Study et son directeur Björn Wittrock, ainsi que l'Institut de littérature de l'Université d'Uppsala et sa directrice Ann Öhrberg, qui ont accueilli successivement mes recherches sur Thunberg.

2. Pour une présentation détaillée de la carrière de Thunberg, voir Marie-Christine SKUNCKE, CARL PETER THUNBERG, BOTANIST AND PHYSICIAN : CAREER-BUILDING ACROSS THE OCEANS IN THE EIGHTEENTH CENTURY, UPPSALA, SWEDISH COLLEGIUM FOR ADVANCED STUDY, 2014.

3. Voir L. C. ROOKMAAKER \& INGVAR SVANBERG, «BIBLIOGRAPHY OF CARL PETER THUNBERG (1743-1828)», SVENSKA LINNÉSÄLLSKAPETS ÅRSSKRIFT, 1992-1993, P. 7-71.

La Révolution française, 13 | 2018 
4. Andreas ÖNNERfors, «LES VOYAGES DE THUNBERG EN ALLEMAND: LA NAISSANCE LABORIEUSE D'UNE TRADUCTION ", À PARAîTRE DANS PATRICE BRET, JEANNE PEIFFER (DIR.), LA TRADUCTION COMME DISPOSITIF DE COMMUNICATION DANS L'EUROPE MODERNE, PARIS, ÉDITIONS HERMANN, HISTOIRE DES SCIENCES, SOUS PRESSE (ÉTUDE DANS LE CADRE DU PROJET « CARL PETER THUNBERG, EUROPE AND JAPAN », VOIR NOTE 1).

5. Sur l'importance du latin en botanique au XVIII ${ }^{\mathrm{E}}$ SIÈCLE, VOIR FRANÇOISE WAQUET, LE LATIN OU L'EMPIRE D'UN SIGNE, XVII-XXE SIÈCLE, PARIS, ALBIN MICHEL, 1978, P. 116-117.

6. Flora Japonica, Lipsiae, J. G. Müller, 1784. Flora Capensis [...], vol. I, fasc. 1-3, Upsaliae, 1807, 1811 et 1813 (publié probablement à compte d'auteur, imprimé par l'imprimerie de l'université d'Uppsala) ; Flora Capensis [...], Hafniae, Gehrhard Bonnier, vol. II, fasc. 1-2, 1818 et 1820, et vol. I, 1824 ; Flora Capensis [...], [réd.] J. A. Schultes, Stuttgardtiae, J. G. Cotta, 1823.

7. Mémoires de l'Académie des sciences de Stockholm (K. Vetenskaps Academiens Handlingar / Nya Handlingar, sigle $\mathrm{KVAH})$ : plus de 60 articles en suédois. Saint-Pétersbourg: par exemple «Fumariae quatuor novae species e Regno Japonico [...]», Nova Acta Academiae Scientiarium Imperialis Petropolitanae, 12, 1801, p. 101-104 (selon Rookmaaker \& Svanberg 1992-1993, p. 47). Cannelle : « Anmärkningar vid Canelen; gjorde på Ceylon », KVAH (Nya Handlingar), vol. 1, 1780, p. 55-66. Monnaies japonaises : Inträdes-Tal, Om de Mynt-Sorter, Som i äldre och sednare tider blifvit slagne och varit gångbare Uti kejsaredömet Japan [...], Stockholm, K. Vetenskaps-Academien, 1779.

8. Jean Deutz (1743-1784): lettres à Thunberg, 9 mars et 9 octobre 1780, G 300 g, Uppsala Universitetsbibliotek ; voir D. O[nno] WIJNANDS, «THE LETTERS OF MAARTEN HOUTTUYN TO CARL PETTER THUNBERG (1780-1790) », PROCEEDINGS OF THE KONINKLIJKE NEDERLANDSE AKADEMIE VAN WETENSCHAPPEN, 93:1, 1990, P. 78, $82 \mathrm{~N}^{0} 1$. MARTINUS hOUTTUYN (1720-1798): LeTtRe À THUNBERG, 17 SEPTEMBRE 1781, DANS WIJJNANDS 1990, P.81. ARTICLE DE THUNBERG: «BESCHRYVING VAN TWEE NIEUWE SOORTEN VAN PALMBOOMACHTIGE GEWASSEN, UIT JAPAN EN VAN DE KAAP DER GOEDE HOPE [...] ", VERHANDELINGEN, UITGEGEEVEN DOOR DE HOLLANDSCHE MAATSCHAPPYE DER WEETENSCHAPPEN, TE HAARLEM. XX:2, HAARLEM, 1782, P. 419-434.

9. Houttuynia, Wijnands, 1990, p. 85 (KVAH 1783). Société de Vlissingen, Wijnands, 1990, p. 86, 88. Traduction néerlandaise de l'article sur la cannelle (voir ci-dessus note 7): Rookmaaker \& Svanberg, 1992-1993, p. 31 ( $\left.{ }^{\circ} 108,1785\right)$.

10. « Ett kort utdrag af en Journal, hållen på en resa til och uti Kejsaredömet Japan, gjord af Doctor Thunberg åren 1775 och 1776, skrifvit til Herr Joseph Banks, Præses uti Royal Society, i London", et traduction anglaise, "Translation of a short Extract from a Journal kept by $C$. p. Thunberg, M. D. during his Voyage to, and Residence in, the Empire of Japan, in a Letter addressed to the President ", Philosophical Transactions, vol. LXX, part I, London, Royal Society, 1780, p. 143-156 (en suédois) ; Appendix, p. i-viii (en anglais). Sur les relations entre Thunberg et le cercle de Joseph Banks, voir SKUNCKE, CARL PETER THUNBERG, OP. CIT., P. 176-185, 197-198.

11. Journal encyclopédique ou universel, $1^{\mathrm{er}}$ octobre 1781, VII:1, p.111-119. Esprit des journaux, novembre 1781, p. 223-232. "Das Neueste von Japan", dans Taschenbuch zum Nutzen und Vergnügen fürs Jahr 1782, Göttingen [1781], p. 1-15, d'après une édition fac-similé du Göttinger Taschen-Calender pour l'année 1782, Mainz, Dieterisch'sche Verlagsbuchhandlung, 1995. Voir SKUNCKE, CARL PETER THUNBERG, OP. CIT., P. 200-201.

12. Resa uti Europa, Africa, Asia, förrättad Åren 1770-1779, 4 vol., Upsala [à compte d'auteur], 1788-1793. Pour une présentation générale, voir SKUNCKE, CARL PETER THUNBERG, OP. CIT., P. 253-257, ET CARL JUNG, KAROSS UND KIMONO. 'HOTTENTOTTEN' UND JAPANER IM SPIEGEL DES REISEBERICHTS VON CARL PETER THUNBERG (1743-1828), (DISS. ZÜRICH), STUTTGART, FRANZ STEINER VERLAG, 2002.

13. Sur le Japon et l'Europe, voir par exemple Jacques PROUST, L'EUROPE AU PRISME DU JAPON, XVII-XVIII SIÈCLE, PARIS, ALBIN MICHEL, 1997.

14. Engelbert KAEMPFER, GESCHICHTE UND BESCHREIBUNG VON JAPAN, HRSG. CHRISTIAN WILHELM DOHM, 2 VOL., LEMGO, 1777-1779 (ÉDITION FAC-SIMILÉ, STUTTGART, 1964).

15. Roger CHARTIER, « LES LIVRES DE VOYAGE », DANS HENRI-JEAN MARTIN ET ROGER CHARTIER (DIR.), HISTOIRE DE L'ÉDITION FRANÇAISE, II. LE LIVRE TRIOMPHANT, 1660-1830, PARIS, PROMODIS, 1984, P. 216. 
16. ÖNNERFORS, «LES VOYAGES DE THUNBERG EN ALLEMAND », OP. CIT. TRADUCTIONS ALLEMANDES : REISEN IN AFRIKA UND ASIEN, VORZÜGLICH IN JAPAN [...], AUSZUGSWEISE ÜBERSETZT VON KURT SPRENGEL [...] UND MIT ANMERKUNGEN BEGLEITET VON JOHANN REINHOLD FORSTER, BERLIN, VOSS, 1792, ET REISE DURCH EINEN THEIL VON EUROPA, AFRIKA UND ASIEN, HAUPTSÄCHLICH IN JAPAN, IN DEN JAHREN 1770 BIS 1779, FREY ÜBERSETZT VON CHRISTIAN HEINRICH GROSKURD, 2 VOL., BERLIN, HAUDE UND SPENER, 1792-1794.

17. SKUNCKE, CARL PETER THUNBERG, OP. CIT., P. 260-262. TRAVELS IN EUROPE, AFRICA, AND ASIA PERFORMED BETWEEN THE YEARS 1770 AND 1779, 4 VOL., LONDON, W. RICHARDSON \& J. EGERTON, VOL. 1-3, [1793] ; F. \& C. RIVINGTON, VOL. $4,1795$.

18. Voyage en Afrique et en Asie, principalement au Japon [...] : Servant de suite au voyage de D. [sic] Sparmann, Paris, Fuchs, an III [1794]. Voyages au Japon, Par le Cap de Bonne-Espérance, les îles de la Sonde, \&c, Paris, Benoît Dandré, an IV [1796], deux éditions, 4 vol. in- $8^{\circ}$ et 2 vol. in $-4^{\circ}$.

19. Benoît d'André (Dandré) à Thunberg, G 300 b, Uppsala Universitetsbibliotek. Les dates de naissance et de mort de d'André sont inconnues - e-mail de Sabine Juratic à l'auteure, 4 décembre 2013.

20. D’André à Thunberg, 21 novembre 1791. Félix Vicq d'Azyr : Nouvelle biographie générale : depuis les temps les plus reculés jusqu'à nos jours, Paris, Firmin Didot frères, t. 46, 1866, col. 89-91.

21. Adresses à Paris : d'André à Thunberg, 18 mai 1792 (Ministre de l'Intérieur Roland, pour Vicq d'Azyr), 18 novembre 1792 (Ministre Lebrun). Consul Delisle (ou De l'Isle), 18 novembre 1792 et passim. Sur Yves Joseph Noël Clemenceau de Lisle (1727-1821), en poste en Suède de 1767 à 1800 , voir Anne MÉZIN, LES CONSULS DE FRANCE AU SIÈCLE DES LUMIÈRES (1715-1792), PARIS, MINISTÈRE DES AFFAIRES ÉTRANGÈRES, 1997, P. 205.

22. 10 septembre 1792 .

23. 2 décembre 1793.

24. 12 mars 1792 (Villeneuve); 26 octobre 1792 (150 riksdaler); 11 janvier 1793 (premier volume arrivé); 29 novembre 1793 (deux traducteurs après Villeneuve); 3 et 14 juin 1793 (manuscrit perdu et retrouvé) ; 23 juin 1794 (quatrième volume complet).

25. 12 mars 1792, 14 mai 1792, 10 septembre 1792 (citations tirées de cette dernière lettre).

26. 18 novembre 1792 .

27. 20 janvier 1794 .

28. 29 juin 1792.

29. 20 janvier et 7 février 1794 ; voir aussi 10 septembre 1792, 30 avril 1793.

30. 29 novembre 1793 et 20 janvier 1794 .

31. Deux lettres sans date [printemps 1795].

32. 18 mai 1792, 29 juin 1792. Voir aussi 21 novembre 1791, sur C. G. Murr à Nuremberg, et, sur Murr, ÖNNERFORS, «LES VOYAGES DE THUNBERG EN ALLEMAND », OP. CIT.

33. Voyage en Afrique et en Asie, 1794, Préface. Anders SPARRMAN, RESA TILL GODA HOPPS-UDDEN, SÖDRA POL-KRETSEN OCH OMKRING JORDKLOTET, SAMT TILL HOTTENTOTT- OCH CAFFER-LANDEN, ÅREN 1772-76, VOL. I, STOCKHOLM, 1783. TRADUCTION FRANÇAISE PAR PIERRE LE TOURNEUR : VOYAGE AU CAP DE BONNE-ESPÉRANCE ET AUTOUR DU MONDE AVEC LE CAPITAINE COOK, ET PRINCIPALEMENT DANS LE PAYS DES HOTTENTOTS ET DES CAFFRES, PAR ANDRÉ SPARRMAN, PARIS, BUISSON, 1787, 2 VOL.

34. Voyage en Afrique et en Asie, 1794, Kaempfer, p. 442-448. Georgi : p. 425-442.

35. D'André à Thunberg, 12 septembre et 9 novembre 1795.

36. Voyages de C. P. Thunberg..., 1796 ; outre «BENOÎT DANDRÉ, LIBRAIRE-ÉDITEUR », LA PAGE DE TITRE INDIQUE LES LIBRAIRES PARISIENS GARNERY ET OBRÉ. LA BIBLIOTHÈQUE UNIVERSITAIRE D'UPPSALA POSSÈDE UN EXEMPLAIRE DE L'ÉDITION IN-OCTAVO, SUR LAQUELLE SE BASENT MES RÉFÉRENCES ; LA BIBLIOTHÈQUE ROYALE DE STOCKHOLM POSSÈDE LES DEUX ÉDITIONS; LA BIBLIOTHÈQUE NATIONALE DE FRANCE POSSÈDE L'ÉDITION INQUARTO, DONT LE PREMIER VOLUME EST EN LIGNE (WWW.GALLICA.BNF.FR).

37. «CAROLO PETRO THUNBERG, / BOTANICES P.P. / Upsalensi, Equiti de Wasa, bene merito » (traduction libre). Portrait dessiné par Notté et gravé par François Denis Née. 
38. Sur Louis-Mathieu Langlès (1763-1824), voir l'article de Frédéric HITZEL DANS DICTIONNAIRE DES ORIENTALISTES DE LANGUE FRANÇAISE DE L'IISMM-KARTHALA, PARIS, ÉD. FRANÇOIS POUILLON, [2008], DEUXIÈME ÉDITION, 2010, P. 559. SELON JEAN-LUC CHAPPEY (E-MAIL À L'AUTEUR, 16 JUILLET 2013), LA PUBLICATION DES VOYAGES DE THUNBERG POURRAIT PARTICIPER DE LA STRATÉGIE DE LANGLÈS POUR JUSTIFIER ET LÉGITIMER SON ACCESSION À LA DIRECTION DE LA BIBLIOTHÈQUE NATIONALE EN OCTOBRE 1795.

39. Voyages de C. P. Thunberg..., op. cit., vol. I, p. iv-v.

40. Sections ajoutées: Java, vol. II, p. 288-323 (sur la langue «javane», vol. II, p. 292-307); "Traité des monnaies, poids et mesures du Japon », vol. IV, p. 359-386 (une traduction allemande du discours suédois a pu servir d'intermédiaire, voir SKUNCKE, CARL PETER THUNBERG, OP. CIT., P. 199).

41. Sources : Préface du Rédacteur, vol. I, p. x-xi. Références abondantes dans les notes. " Notice alphabétique et raisonnée des ouvrages cités dans les Notes du Rédacteur », vol. IV, p. 417-462.

42. Préface, vol. I, p. i-ii et vi-x.

43. Préface, vol. I, p. iii-iv.

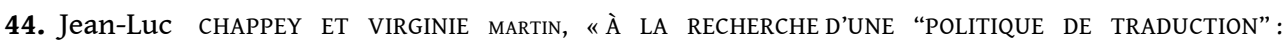
TRADUCTEURS ET TRADUCTIONS DANS LE PROJET RÉPUBLICAIN DU DIRECTOIRE (1795-1799) », LA RÉVOLUTION FRANÇAISE [EN LIGNE], 12 | 2017, HTTP://JOURNALS.OPENEDITION.ORG/LRF/1732, (MIS EN LIGNE LE 15 SEPTEMBRE 2017, CONSULTÉ LE 27 NOVEMBRE 2017).

45. Langues orientales : vol. III, p. 2-3, note. Anckarström : vol. I, p. 71, note.

46. Thunberg à André Thouin, 11 novembre 1796, Ms. 1989, Muséum d'histoire naturelle, Paris.

47. Pour le débat sur Linné en France au début de la République française, voir Jean-Luc CHAPPEY, DES NATURALISTES EN RÉVOLUTION. LES PROCÈS-VERBAUX DE LA SOCIÉTÉ D'HISTOIRE NATURELLE DE PARIS (1790-1798), PARIS, ÉDITIONS DU CTHS, 2009, P. 18-37. SUR LAMARCK BOTANISTE, VOIR PAR EXEMPLE PASCAL DURIS, LINNÉ ET LA FRANCE (1780-1850), GENÈVE, DROZ, 1993, P. 146-151; SUR SON RÔLE DANS LA CONTROVERSE LINNÉ-BUFFON, VOIR PIETRO CORSI, LAMARCK. GENÈ̇E ET ENJEUX DU TRANSFORMISME, 1770-1830, PARIS, CNRS ÉDITIONS, 2001.

48. Citoyen Lamarck, « Explication des figures » [d'histoire naturelle], vol. IV, p. 348-358.

49. Sur le «Rangaku », voir par exemple Jacques PROUST, L'EUROPE AU PRISME DU JAPON, OP. CIT., CH. VII ; LEONARD BlusSÉ ET AL. (DIR.), BRIDGING THE DIVIDE: 400 YEARS THE NETHERLANDS - JAPAN, LEIDEN, HOTEI PUBLISHING \& HILVERSUM, TELEAC/NOT, 2000, CH. 5-7 (SUR PH. F. VON SIEBOLD, 1796-1866, P. 113, ARTICLE DE HARM BEUKERS).

50. Sur von Siebold, Itō Keisuke (1803-1901), et le Taisei Honzō Meiso (Nagoya [1829] ), y compris le portrait de Thunberg, voir l'analyse de Richard C. RUDOLPH, «THUNBERG IN JAPAN AND HIS FLORA JAPONICA IN JAPANESE », MONUMENTA NIPPONICA, VOL. 29, NO. 2, 1974, P. 172-178 ; SKUNCKE, CARL PETER THUNBERG, OP. CIT., P. 274-282. THUNBERG SOUS LA RESTAURATION MEIJI : YOJIRŌ KIMURA, «THUNBERG AND THE FLORA OF JAPAN », DANS C. P. THUNBERG'S DRAWINGS OF JAPANESE PLANTS : ICONES PLANTARUM JAPONICARUM THUNBERGII [...], [RÉD.] Y. KIMURA \& V. P. LEONOV, TOKYO, MARUZEN CO., 1994, P. 333 (« THE BASIC REFERENCE FOR BOTANICAL RESEARCH »).

51. Sur Greifswald et la Poméranie suédoise, voir l'étude d'Andreas ÖNNERFORS, CI-DESSUS, NOTE 4.

\section{RÉSUMÉS}

Le botaniste et médecin suédois Carl Peter Thunberg (1743-1828) est le seul savant européen à avoir visité et décrit le Japon au XVIII ${ }^{\mathrm{e}}$ siècle. Ses ouvrages, traduits en plusieurs langues, ont connu une large diffusion en Europe. La première partie de la présente étude aborde ses 
monographies scientifiques en latin, dont la Flora Japonica (1784), ainsi que ses articles, publiés tantôt en latin, tantôt en langue vernaculaire. La deuxième partie est consacrée aux traductions européennes de son récit de voyage dans les années 1790, mettant l'accent sur les versions françaises. Une série de lettres manuscrites envoyées à Thunberg à Uppsala par Benoît Dandré (ou d'André), éditeur-libraire à Paris pendant la Révolution, permet de suivre de près la genèse de l'édition française de 1796. La troisième partie de l'article évoque brièvement une adaptation japonaise de la Flora Japonica, le Taisei Honzō Meiso d'Itō Keisuke, (1829), qui fit connaître le botaniste suédois au Japon.

The Swedish botanist and physician Carl Peter Thunberg (1743-1828) was the only European scientist who visited and published his observations of Japan in the eighteenth century. His works were translated into several European languages. The first part of this article is devoted to the diffusion of his scientific monographs in Latin (including the Flora Japonica, 1784) and that of his articles, published either in Latin or vernacular languages. The second part is devoted to the European translations of his travel account, concentrating on the French versions. A series of manuscript letters to Thunberg from his publisher in Paris during the French Revolution, the bookseller Benoît Dandré (or d'André), makes it possible to follow in detail the genesis of the French 1796 edition. The third part briefly presents a Japanese adaptation of Thunberg's Flora Japonica, Itō Keisuke's Taisei Honzō Meiso (1829), which made the Swedish botanist famous in Japan.

\section{INDEX}

Keywords : Translation, Carl Peter Thunberg, Japan, natural history, travel literature

Mots-clés : Traduction, Carl Peter Thunberg, Japon, histoire naturelle, récit de voyage

\section{AUTEUR}

\section{MARIE-CHRISTINE SKUNCKE}

Institut de littérature, Université d'Uppsala. 